\title{
Conduction Cooling of a Niobium SRF Cavity Using a Cryocooler
}

\author{
Joshua Feldman ${ }^{1,2}$, Michael Geelhoed ${ }^{2}$, Ram Dhuley ${ }^{2}$ Jayakar C. Thangaraj ${ }^{2}$ \\ ${ }^{1}$ University of Illinois at Urbana-Champaign, Urbana, IL, USA \\ ${ }^{2}$ Illinois Accelerator Research Center (IARC), Fermi National Accelerator Laboratory, Batavia, IL, USA
}

\begin{abstract}
Superconducting Radio Frequency (SRF) cavities are the primary choice for accelerating charged particles in high-energy research accelerators. Institutions like Fermilab use SRF cavities because they enable significantly higher gradients and quality factors than normal-conducting RF cavities and DC voltage cavities. To cool the SRF cavities to low temperatures (typically around $2 \mathrm{~K}$ ), liquid helium refrigerators are used. Producing and maintaining the necessary liquid helium requires large, elaborate cryogenic plants involving dewars, compressors, expansion engines, and recyclers. The cost, complexity, and space required for such plants is part of the reason that industry has not yet adopted SRF-based accelerators. At the Illinois Accelerator Research Center (IARC) at Fermilab, our team seeks to make SRF technology accessible not only to large research accelerators, but to industry as well. If we eliminate the complexity associated with liquid helium plants, SRF-based industrial accelerators may finally become a reality. One way to do this is to eliminate the use of liquid helium baths altogether and develop a brand-new cooling technique for SRF cavities: conduction cooling using a cryocooler. Recent advances in SRF technology have made it possible to operate SRF cavities at $4 \mathrm{~K}$, a temperature easily achievable using commercial cryocoolers. Our IARC team is taking advantage of this technology to cool SRF cavities.
\end{abstract}

\section{Introduction and motivation}

From sewage water irradiation to medical equipment sterilization to electron beam welding, particle accelerators are a hallmark of modern society. SRF cavities enable accelerators to produce far more powerful and efficient accelerator beams than most other methods. If SRF cavities could be used economically in industrial applications, it would enable more powerful industrial accelerators as well as many new possible industrial applications. However, producing and maintaining the liquid helium required to cool SRF cavities is currently not very economical for industrial accelerators, partly due to the complex cryogenic plants required. Not only do they take up a lot of space, but due to their complexity, maintenance and operation

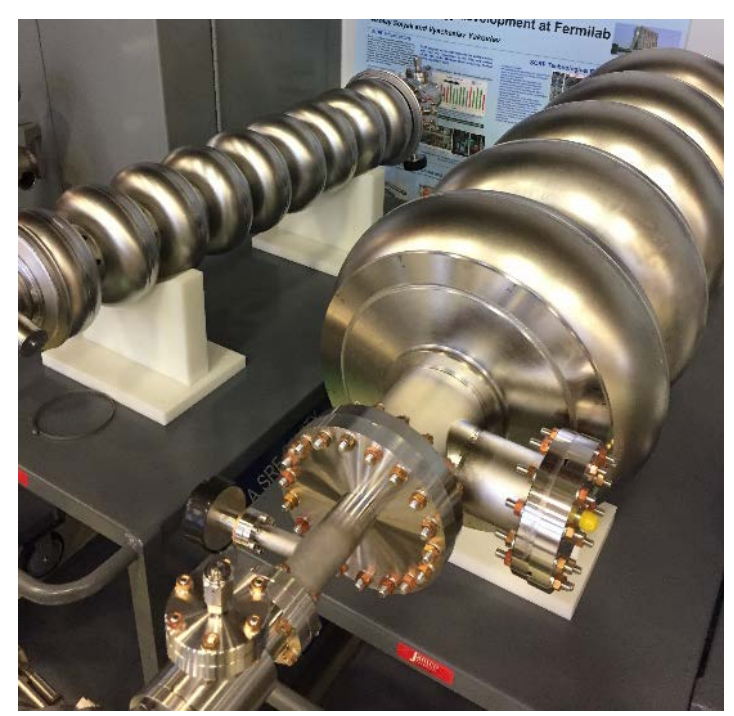

Figure 1: Niobium SRF cavities on display at Fermilab 
of these plants often requires experts on hand. Furthermore, there is a significant monetary cost associated with the construction of the plants and the electrical power needed to operate them. The need for cryogenic plants in producing liquid helium is one of the reasons that there are no SRF-based industrial accelerators in operation today. At IARC, we believe that if we could develop a new technique for cooling an SRF cavity that does not require liquid helium, SRFbased accelerators may then become economical for industrial applications and not just research applications. The new technique

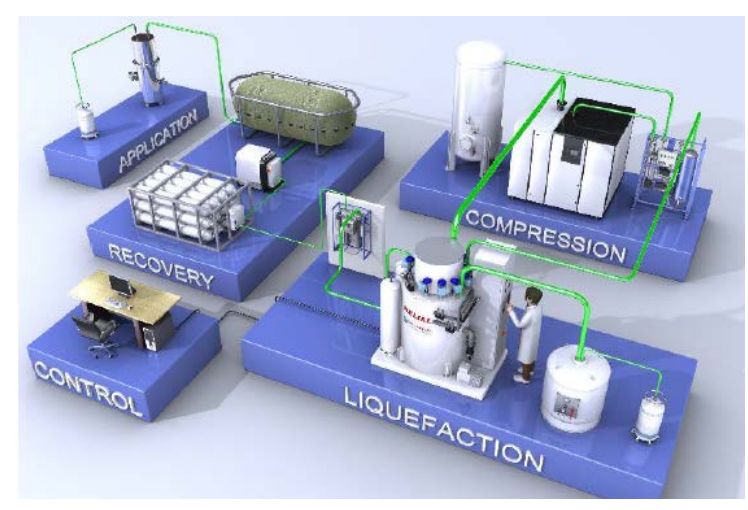

Figure 2: A typical helium liquefaction plant [1]. A man is shown to depict the size and complexity of such plants

we are exploring is conduction cooling an SRF cavity using a commercially available cryocooler. By cooling an SRF cavity with a cryocooler, the infrastructure needed for an SRF accelerator is radically simplified, saving a lot of space and money. For example, rather than needing a large, complex network of compressors, expansion engines, and dewars, a cryocooler cold head and compressor are all that are needed. Cryocooler equipment, unlike liquid helium liquefaction equipment, may be portable, enabling portable accelerators for new, innovative uses.

Cryostats designed for conduction cooling will be simpler than those for liquid helium baths. Current SRF cryostats have complex designs that must carefully control pressure, leakage, and evaporation. A future conduction cooling cryostat need only to maintain a proper vacuum. Furthermore, a cryocooler apparatus does not require as much expertise to operate and maintain than a liquid helium liquefaction plant, easing the operation of SRF accelerators. Such a compact and simple accelerator would find innovative new uses in industry, medicine, security, and the environment, possible creating entirely new industries. For example, a compact conduction-cooled SRF accelerator could be truck-mounted and used to treated newly-paved highway. SRF electron accelerators could replace Cobalt 60, a dangerous radioactive isotope, as a source of radiation. They could catalyze chemical reactions to save time and energy, or they could irradiate sewage water. Some of these applications are currently being evaluated at Fermilab.

While our aim is to cool an SRF cavity using a cryocooler, we are not yet sure how to do so. Fermilab is the first to attempt conduction cooling of a Niobium SRF cavity using a cryocooler, so we have to start from scratch and develop a cooling mechanism. This brings along several difficult engineering challenges. Even state-of-the-art cryocoolers can only remove approximately 1-2 watts of heat at temperatures around $4.2 \mathrm{~K}$ [2], while SRF cavities are expected to dissipate 1-2 watts of heat at $4.2 \mathrm{~K}$. Therefore, our method of connecting a cavity to a cryocooler must allow for excellent heat flow, or it will not work. This is our challenge.

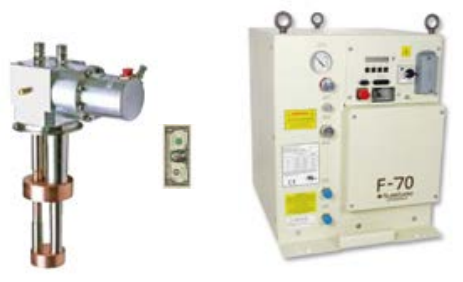

Figure 3: A typical cryocooler setup [2]. Dollar shown for scale. 


\section{Challenge: Design of cooling and support Structures}

We must engineer a structure through which heat can effectively be removed from the cavity to the cryocooler. Through 3D modeling, we drafted a variety of structures that would thermally and mechanically connect the cavity to the cryocooler cold head. All of these concept structures are composed of high purity $(5 \mathrm{~N})$ aluminum (high purity aluminum has an outstanding thermal conductivity at temperature around $4.2 \mathrm{~K}$, making it an obvious candidate for an application like this).

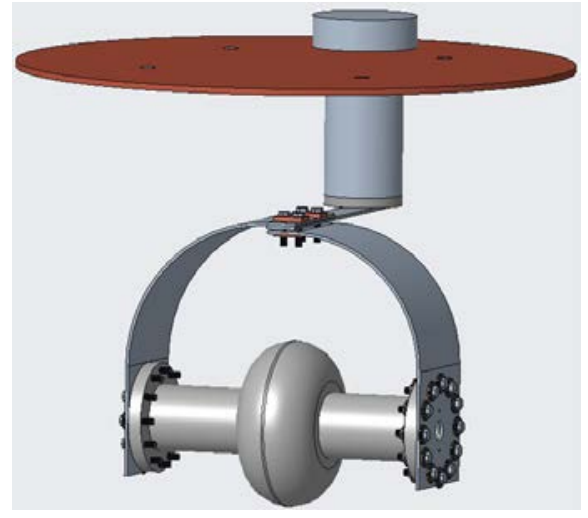

Figure 4: A concept model for cooling a

$1.3 \mathrm{GHz}$ niobium SRF cavity

Shown here is one such draft (Figure 4). In this model, aluminum arcs connect the cavity flange to an aluminum strip, which then connects to the cryocooler cold head. All mechanical joints are bolted together. Designing such models requires significant geometric analysis to align all the components properly.

Cryocoolers themselves cannot support the weight of heavy cavities, so we had to design a way to support a cavity's weight inside a cryostat. An ideal support structure would hold a cavity rigidly in its place while keeping it completely thermally isolated from its surroundings. Again, 3D modeling was central to our design process. One support model (Figure 5 ) is shown here. We began this process by using G-10 Garolite rods. These rods are mounted to

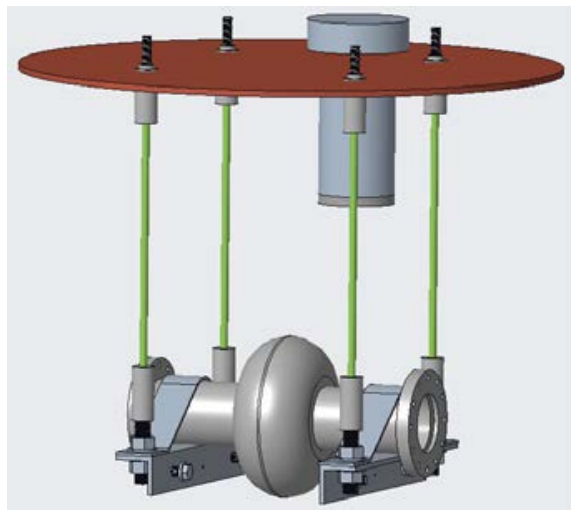

Figure 5: A concept model for supporting a $1.3 \mathrm{GHz}$ niobium SRF cavity

the $60 \mathrm{~K}$ copper plate portion of the cryocooler, and prevent heat flow into the cavity as they have very poor thermal conductivity. G-10 also has excellent structural strength, suiting it to supporting a heavy load. The cavity then rests on Aluminum L-brackets, which are thermally isolated from the rods via stacked nylon washers. The $L$ shape provides rigidity and the Aluminum cools quickly to prevent any heat load on the cavity. It was difficult to keep the cavity securely in place while using a little material as to prevent a heat load.

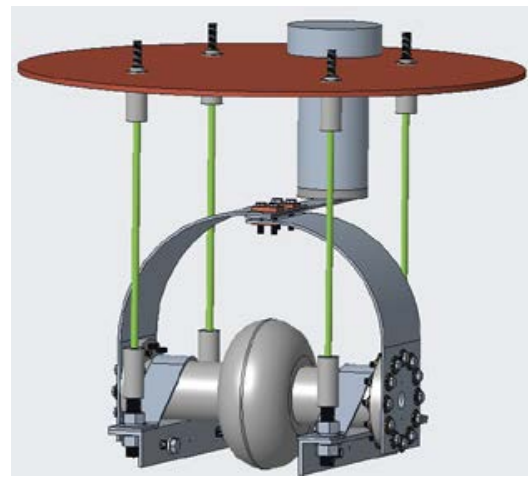

Figure 6: The integrated setup

All the parts that you see in these 3D models have been machined, assembled, and are currently being tested in a cryostat at Fermilab (Figure 8). 


\section{Challenge: Optimizing Thermal Connections}

The contact points between two metal components are likely to dominate the total thermal resistance of the cooling structure. These contact points may be between the niobium cavity surface and the high-purity aluminum, or between two aluminum components. This is because, at a microscopic level, the contact between two components is simply the incidental contacts between the jagged surfaces of the two metals. The thermal resistance at these joints is believed to be significant enough to prevent us from effectively cooling the cavity, so we have directed significant $R$ \& $D$ efforts at minimizing the thermal resistances at these joints. To minimize these resistances, we tested three different variables: interposer, bolting pressure, and surface preparation. To measure the thermal resistance across the joint, we used the twoheater one-thermometer method [3]. This method, shown in Figure 6, consists of placing an "upstream" heater, a "downstream" heater, and an "upstream" thermometer. We measure the steady-state temperature with the upstream heater on, $T_{1}$, then the steady temperature with the downstream heater on, powered with the same heat $Q$ as the upstream heater, $T_{2}$, and divide the difference by the heat flow to get thermal resistance.

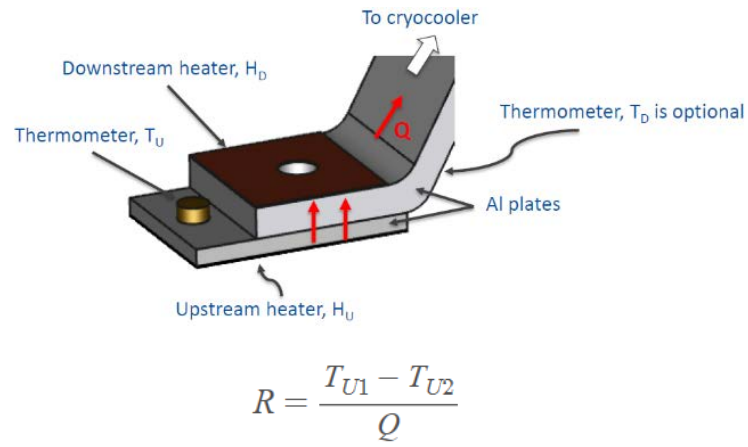

Figure 7: An explanation of the twoheater one-thermometer method for measuring thermal resistance [3]

\section{Progress and Future Plans}

The bulk of my work this summer has been focused on engineering the cooling and support structures and optimizing thermal connections. However, my team and I have made real progress towards cooling an actual cavity. We are currently cooling down a $1.3 \mathrm{GHz}$ Niobium SRF cavity at IARC, using the cooling and support structures I helped design. The goal of this cooling experiment is to gauge the cool-down time for a $1.3 \mathrm{GHz}$ cavity, as well as test the viability of both the designed structures.

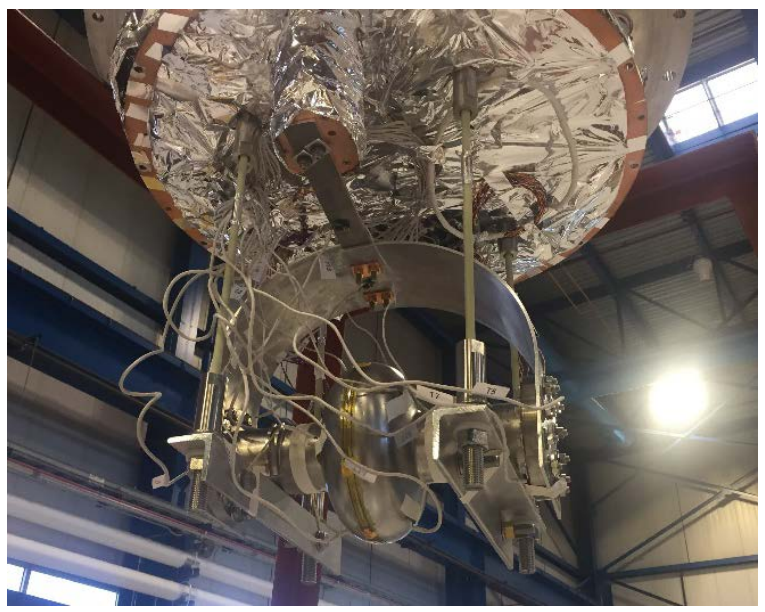

Figure 8: Our first conduction-cooling experiment of a $1.3 \mathrm{GHz}$ niobium SRF cavity

Furthermore, we are currently automating our experimentation. Using LabVIEW software and a variety of electronics, we are enabling our thermometry and heater system to be remotely monitored and controlled by a computer. This saves our time precious time as we will not have to remain on-location as the experiment is going on, freeing up time to do other work. We have also automated our vacuum readout system. Now, our lab computer will send out email alert notifying one of us that it is time to turn on the turbo pump stage of the vacuum process, or that it is time to turn on the cryocooler. This expedites our cool-down process as we will have a convenient way of knowing exactly when to turn on the cryocooler, saving us time. 


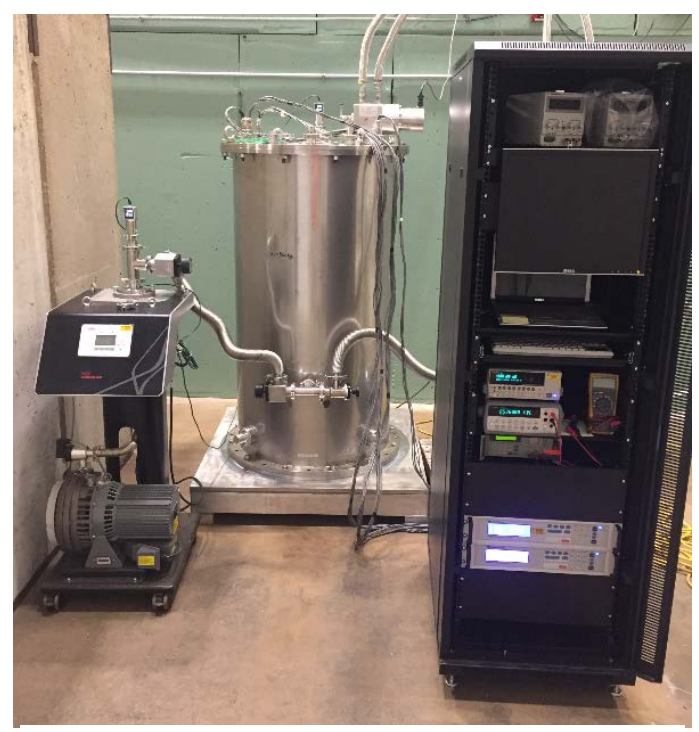

Figure 9: Our experimental setup includes a vacuum pump (left), cryostat (right), and instrumentation rack (right)

Finally, we have spent time using 3D-modeling to explore a variety of alternative cooling and support structures, ones that we believe may have better thermal properties than the designs displayed in Section II. We have prepared a variety of engineering drawings and are preparing to test these alternative structures as a viable option.

\section{Acknowledgements}

Fermi National Accelerator Laboratory is operated by Fermi Research Alliance, LLC under Contract No. DE-AC02-07CH11359 with the United States Department of Energy.

\section{References}

[1] R. Kephart, et al., "SRF AND COMPACT ACCELERATORS FOR INDUSTRY AND SOCIETY" Proceedings of SRF2015, Whistler, BC, Canada, FRBA03

[2] SHI Cryogenics Group, "Cryocooler Product Catalogue", 9, 162017

[3] R.C. Dhuley, et al. "Thermal conductance characterization of a pressed copper rope strap between $0.13 \mathrm{~K}$ and $10 \mathrm{~K}$ ", Cryogenics 86, 17-

21, 2017 